\title{
Forecasting Value-at-Risk Using Block Structure Multivariate Stochastic Volatility Models*
}

\author{
Manabu Asai \\ Faculty of Economics \\ Soka University \\ Japan \\ Massimiliano Caporin \\ Department of Economics and Management "Marco Fanno" \\ University of Padova \\ Italy \\ Michael McAleer \\ Econometric Institute \\ Erasmus University Rotterdam \\ Erasmus School of Economics \\ and \\ Tinbergen Institute \\ The Netherlands \\ and \\ Department of Quantitative Economics \\ Complutense University of Madrid \\ and \\ Institute of Economic Research \\ Kyoto University
}

Revised: March 2012

*The third author is grateful for the financial support of the Australian Research Council, National Science Council, Taiwan, and the Japan Society for the Promotion of Science. 


\begin{abstract}
Most multivariate variance or volatility models suffer from a common problem, the "curse of dimensionality". For this reason, most are fitted under strong parametric restrictions that reduce the interpretation and flexibility of the models. Recently, the literature has focused on multivariate models with milder restrictions, whose purpose was to combine the need for interpretability and efficiency faced by model users with the computational problems that may emerge when the number of assets is quite large. We contribute to this strand of the literature proposing a block-type parameterization for multivariate stochastic volatility models. The empirical analysis on stock returns on US market shows that $1 \%$ and $5 \%$ Value-at-Risk thresholds based on one-step-ahead forecasts of covariances by the new specification are satisfactory for the period includes the global financial crisis.
\end{abstract}

Keywords: block structures; multivariate stochastic volatility; curse of dimensionality; leverage effects; multi-factors; heavy-tailed distribution.

JEL classifications: C32, C51, C10. 


\section{Introduction}

Classical portfolio allocation and management strategies are based on the assumption that risky returns series are characterized by time invariant moments. However, the econometric literature of the last few decades demonstrated the existence of dynamic behaviour in the variances of financial returns series. The introduction of such empirical evidence may constitute an additional source of performance for portfolio managers, as evidenced by Fleming, Kirby and Ostdiek (2001), or may be relevant for improving the market risk measurement and monitoring activities (see, for example, Hull and White (1998) and Lehar et al. (2002)). Two families of models emerged in the literature, namely GARCH-type specifications (see Engle (2002)), and Stochastic Volatility models (see Taylor (1986) and Andersen (1994)).

However, portfolio management strategies often involve a large number of assets requiring the use of multivariate specifications. Among the possible alternative models, we cite the contributions of Bollerslev (1990), Engle and Kroner (1995), Ling and McAleer (2003), Asai and McAleer (2006, 2009a,b), and the surveys in McAleer (2005), Bauwens, Laurent and Rombouts (2006), Asai, McAleer and Yu (2006) and Chib, Omori and Asai (2009). Most models, if not all, suffer from a common problem, the well-known "curse of dimensionality", whereby models become empirically infeasible if fitted to a number of series of moderate size (in some cases, the models may become computationally intractable with even 5 or 6 assets). In order to match the need of introducing time-varying variances with practical computational problems, several restricted models are generally used: the diagonal VECH specifications suggested by Bollerslev, Engle and Wooldridge (1988), the scalar VECH and BEKK models proposed 
by Ding and Engle (2001), the CCC model of Bollerslev (1990), and the dynamic conditional correlation models of Engle (2002) and Tse and Tsui (2002). However, the introduction of significant and strong restrictions reduces the interpretation and flexibility of the models, possibly affecting the purportedly improved performance they may provide and/or the appropriateness of the analysis based on their results.

Recently, the literature has focused on multivariate models with milder restrictions, whose purpose was to combine the need for interpretability and efficiency faced by model users with the computational problems that may emerge when the number of assets is quite large. Among the contributions in this direction, we follow the approach of Billio, Caporin and Gobbo (2006). They proposed specifying the parameter matrices of a general multivariate correlation model in a block form, where the blocks are associated with assets sharing some common feature, such as the economic sector. Our purpose is to adopt this block-type parameterization and adapt it to multivariate stochastic volatility models.

In general terms, Multivariate Stochastic Volatility (MSV) models have a parameter number of order $O\left(M^{2}\right)$, where $M$ is the number of assets. With the introduction of block parameter matrices, we may control the number of parameters and obtain a model specification which is feasible, even for a very large number of assets. Furthermore, as in the contribution of Billio, Caporin and Gobbo (2006), the models we propose follow the spirit of sectoral-based asset allocation strategies since they will presume the existence of common dynamic behaviour within assets or financial instruments belonging to the same economic sector. This assumption is not as strong as postulating 
the existence of a unique factor driving all the variances and covariances, since the financial theory may suggest the existence of sector-specific risk factors (sectoral asset allocation is often followed by portfolio managers and characterized by a number of managed financial instruments).

As distinct from an extremely restricted model, we also recover part of the spillover effect between variances, which allows monitoring of the interdependence between groups of assets, an additional element which may be relevant. Within our modeling approach, the coefficients may be interpreted as sectoral specific, while the assets will be in any case characterized by a specific long term variance through the introduction of unrestricted constants in the variance equations.

For the purpose of explaining our approach, we consider a multi-component MSV model allowing leverage effect and heavy-tailed unconditional distribution, which is a multivariate extension of Chernov et al. (2003), although our approach is applicable to the factor model of Pitt and Shephard (1999) and Chib, Nardari, and Shephard (2006) and the dynamic correlation model of Asai and McAleer (2009b).

Clearly, the restrictions proposed may not necessarily be accepted by the data, as more 'complete' models will, in general, provide better results. We will show that the introduction of such restrictions provides limited losses, while yielding a significant improvement over the more restricted specifications. We will evaluate and compare the out-of-sample forecast of alternative models. 
The plan of the remainder of the paper is as follows. Section 2 presents the multi-component MSV models, and discusses the differences between the MSV model and the factor specifications. Section 3 introduces the block-structure modelling approach, and addresses some estimation issues. Section 4 presents an empirical example regarding the out-of-sample forecasts, based on US stock market data for selected firms. Section 5 gives some concluding comments.

\section{Multi-Component MSV Model}

The block-structure model, which we will present in the next section, can be considered as a restricted specification of a general MSV model. In fact we will show how the modelling approach consists in defining a set of parametric restrictions that makes the model feasible, but without losing the interpretation of coefficients.

We start from the basic MSV model suggested by Harvey, Ruiz and Shephard (1994).

Let $R_{t}$ be the $M$-dimensional vector of asset returns, and define $y_{t}=R_{t}-E\left(R_{t} \mid \Im_{t-1}\right)$, where $\mu_{t}$ is the $M$-dimensional vector of conditional means. Then, the mean equation of the basic MSV model is defined by

$$
\begin{gathered}
y_{t}=D_{t} \varepsilon_{t}, \\
D_{t}=\operatorname{diag}\left\{\exp \left(0.5 h_{t}\right)\right\},
\end{gathered}
$$

where $h_{t}$ is the $M$-dimensional vector of stochastic volatilities, $\exp (x)$ for a vector $x$ 
is the element-by-element operator of exponentiation, $\operatorname{diag}\{x\}$ for a vector $x$ is the operator which creates a diagonal matrix with the diagonal element corresponding to those of $x$, and $\varepsilon_{t}$ follow the multivariate normal distribution with covariance matrix defined later. We exclude the case that $\mu_{t}$ is specified by $h_{t}$ as in Koopman and Uspensky (2002), since it is beyond the scope of our paper. The volatility equation of the model is given by

$$
h_{t+1}=\phi \circ h_{t}+\eta_{t}
$$

where $h_{t}$ is the $M$-dimensional vector of stochastic log-volatilities, the operator 。 denotes the Hadamard (or element-by-element) product, $\phi$ is the $M$-vector of parameters, $\varepsilon_{t}$ and $\eta_{t}$ independently follow the multivariate normal distributions as $\varepsilon_{t} \sim N\left(0, S_{\varepsilon} P_{\varepsilon \varepsilon} S_{\varepsilon}\right) \quad$ and $\quad \eta_{t} \sim N\left(0, S_{\eta} P_{\eta \eta} S_{\eta}\right), \quad S_{x}(x=\varepsilon, \eta) \quad$ are $\quad M \times M$ diagonal matrices of standard deviations, and $P_{x x}(x=\varepsilon, \eta)$ are $M \times M$ correlation matrices. In this specification, the vector of log-volatilities follows the $\operatorname{VAR}(1)$ process. For convenience, we call this type of MSV model the 'basic MSV' model.

Based on the basic MSV model, several authors including Danielsson (1998), Chan Kohn and Kirby (2006), Asai and McAleer (2006, 2009a) and Chib, Omori and Asai (2009) suggested models which accommodate the asymmetric effects. The MSV model with Leverage effects (MSVL) is generally specified as the correlation between the 
disturbances of the mean and volatility equations as, $\left(\varepsilon_{t}^{\prime}, \eta_{t}^{\prime}\right)^{\prime} \sim N(0, S P S)$ with

$$
S=\left(\begin{array}{cc}
S_{\varepsilon} & O \\
O & S_{\eta}
\end{array}\right), \quad P=\left(\begin{array}{cc}
P_{\varepsilon \varepsilon} & P_{\varepsilon \eta} \\
P_{\eta \varepsilon} & P_{\eta \eta}
\end{array}\right),
$$

where $S$ is the diagonal matrix of Standard deviations for $\left(\varepsilon_{t}^{\prime}, \eta_{t}^{\prime}\right)^{\prime}$ and $P$ is the corresponding correlation matrix. As the leverage effects are especially observed for the individual correlation between $\varepsilon_{i t}$ and $\eta_{i t}(i=1,2, \ldots, M)$, we may reduce the number of parameters.

Now, we turn to the feature regarding fat-tails of stock return distribution. Although the models of SV and GARCH families enable the observed series to have heavy-tailed distributions, empirical analysis has shown that assuming a Gaussian conditional distribution is insufficient to describe the tail behaviour of real data (see Liesenfeld and Jung (2000), Chib, Nardari, and Shephard (2002), and Asai (2008, 2009)). For the univariate SV models, these authors suggested several extensions on the heavy-tailed conditional distribution, including the Student $t$ distribution, the generalized error distribution, and the mixture-of-normal distribution. With respect to the multivariate SV model, Harvey, Ruiz and Shephard (1994) work with a multivariate $t$ distribution. Yu and Meyer (2006) made a restriction on the degree-of-freedom parameter such that the parameter for individual variable is specified as the same one, and they mentioned that this formulation was empirically better supported than the formulation in Harvey, Ruiz and Shephard (1994). 
An alternative approach for fat-tails is to employ multifactor models, as proposed in Chernov et al. (2003). One of the contributions of Chernov et al. (2003) is to attain a heavy-tailed return distribution by introducing multi-component, without assuming heavy-tailed conditional distributions. See also Alizadeh, Brandt and Diebold (2002), Asai (2008) and Christoffersen, Jacobs and Wang (2008). Based on the idea for the univariate model, Asai and McAleer (2009a) considered the two component MSVL model. The general $K$-component MSVL model is defined by equations (1), (2) and

$$
\begin{aligned}
& h_{t}=\sum_{i=1}^{K} V_{t}^{(i)} \\
& V_{t+1}^{(i)}=\phi^{(i)} \circ V_{t}^{(i)}+\xi_{t}^{(i)} \quad(i=1,2, \ldots, K),
\end{aligned}
$$

with $\phi^{(i)}$ is $M$-vector of parameters, $\xi_{t}^{\prime}=\left(\xi_{t}^{(1)^{\prime}}, \ldots, \xi_{t}^{(K)^{\prime}}\right)$ and $\left(\varepsilon_{t}^{\prime}, \xi_{t}^{\prime}\right)^{\prime} \sim N(0, S P S)$, where $S$ is the diagonal matrix of standard deviation, $S=\operatorname{diag}\left\{\left(\sigma_{\varepsilon}^{\prime}, \sigma_{\xi}^{(1)^{\prime}}, \ldots, \sigma_{\xi}^{(K)^{\prime}}\right)^{\prime}\right\}$ and $P$ is the correlation matrix constructed by $P=\left(Q^{-1} \circ I_{m}\right)^{-1 / 2} Q^{-1}\left(Q^{-1} \circ I_{m}\right)^{-1 / 2}$ and

$$
P=\left(\begin{array}{cc}
P_{\varepsilon \varepsilon} & \Gamma \\
\Gamma^{\prime} & P_{\xi \xi}
\end{array}\right), \quad \Gamma=\left(\begin{array}{lll}
\Gamma_{1} & \cdots & \Gamma_{K}
\end{array}\right), \quad P_{\xi \xi}=\left(\begin{array}{ccc}
P_{\xi \xi}^{(1)} & & O \\
& \ddots & \\
O & & P_{\xi \xi}^{(K)}
\end{array}\right)
$$

with corresponding correlation matrices, $P^{\varepsilon \varepsilon}$ and $P_{\xi \xi}^{(i)}(i=1,2, \ldots, K)$ and diagonal matrices of leverage effects, $\Gamma_{i}=\operatorname{diag}\left\{\gamma^{(i)}\right\} \quad(i=1,2, \ldots, K)$. The number of 
parameters in the $K$-component MSVL model is $(3 K+1) M+(K+1) M(M-1) / 2$. In the empirical analysis, we employ the two component MSVL (MSVL2C) model as the basics of the new block structure model.

At this stage we should discuss the difference between the MSVL2C model and the popular factor MSV model suggested by Pitt and Shephard (1999). In the literature of MSV models, there are two major approaches for modeling factors. One is based on the volatility factor as in Harvey, Ruiz and Shephard (1994), who introduce latent factors instead of latent volatility processes, in order to describe volatilities using small number of factors. Calvet, Fisher, and Thompson (2006) also suggested volatility factor MSV model with Markov switching factors. In their specification, the number of factors is not necessary less than the dimension of $y_{t}$. The other approach for modeling factors is the mean factor model suggested by Pitt and Shephard (1999), who assume the mean factor to have stochastic volatilities, in addition to those in the conditional distribution of $y_{t}$. Based on the mean factor model, Chib, Nardari, and Shephard (2006) allowed for jumps in the observation model and a fat-tailed $t$-distribution, while Lopes and Carvalho (2007) suggested another general model which nests the models of Pitt and Shephard (1999) and Aguilar and West (2000).

With respect to the two categories, the $K$-component MSVL model is classified as the volatility factor model. Compared with mean factor model of Pitt and Shephard (1999) with $M$-factors, the MSV2C, that is, two-component MSV model without leverage, has the same number of parameters. Unlike the model of Pitt and Shephard (1999), the 
MSVL2C model accommodates the leverage effects. However, we should notice that the mean factor models can control the number of mean factors, implying that it enables to reduce the number of parameters by controlling the number of factors. In the following section, we will develop a new approach which reduces the number of parameters by considering block structures. Our new approach is also applicable to the mean factor model for the volatility structure of the disturbance.

\section{Block Structure Model}

The two-component MSVL (MSVL2C) model has two major advantages to the mean factor model of Pitt and Shephard (1999). One is that it is unnecessary to consider heavy-tailed conditional distribution generally, and the other is that it can incorporate leverage effects to the factors straightforwardly.

Now, we develop a new specification based on a block structure of assets. We assume that the $M$ assets are divided into $B$ groups, with the $j$-th group containing $m_{j}$ assets $\left(M=m_{1}+m_{2}+\cdots+m_{B}\right)$. We define a block structure for the volatility by assuming that each group of assets is characterized by a common parametric behaviour in the volatility equation. Consider equation (5) with restrictions on parameters as 


$$
\begin{gathered}
\phi^{(i)}=\left[\begin{array}{c}
\phi_{1}^{(i)} \boldsymbol{l}_{m_{1}} \\
\phi_{2}^{(i)} \boldsymbol{l}_{m_{2}} \\
\vdots \\
\phi_{B}^{(i)} \boldsymbol{l}_{m_{B}}
\end{array}\right], \quad \sigma_{\xi}^{(i)}=\left[\begin{array}{c}
\sigma_{1}^{(i)} \boldsymbol{l}_{m_{1}} \\
\sigma_{2}^{(i)} \boldsymbol{l}_{m_{2}} \\
\vdots \\
\sigma_{B}^{(i)} \boldsymbol{l}_{m_{B}}
\end{array}\right], \quad \gamma^{(i)}=\left[\begin{array}{c}
\gamma_{1}^{(i)} \boldsymbol{l}_{m_{1}} \\
\gamma_{2}^{(i)} \boldsymbol{l}_{m_{2}} \\
\vdots \\
\gamma_{B}^{(i)} \boldsymbol{l}_{m_{B}}
\end{array}\right], \\
P_{\xi \xi}^{(i)}=\left(\begin{array}{cccc}
P_{\xi \xi, 11}^{(i)} & \rho_{21}^{(i)} \boldsymbol{l}_{m_{2}} \boldsymbol{l}_{m_{1}}^{\prime} & \cdots & \rho_{B 1}^{(i)} \boldsymbol{l}_{m_{1}} \boldsymbol{l}_{m_{B}}^{\prime} \\
\rho_{21}^{(i)} \boldsymbol{l}_{m_{2}} \boldsymbol{l}_{m_{1}}^{\prime} & P_{\xi \xi, 22}^{(i)} & \cdots & \rho_{B 2}^{(i)} \boldsymbol{l}_{m_{2}} \boldsymbol{l}_{m_{B}}^{\prime} \\
\vdots & \vdots & \ddots & \vdots \\
\rho_{B 1}^{(i)} l_{m_{B}} \boldsymbol{l}_{m_{1}}^{\prime} & \rho_{B 2}^{(i)} \boldsymbol{l}_{m_{B}} \boldsymbol{l}_{m_{2}}^{\prime} & \cdots & P_{\xi \xi, B B}^{(i)}
\end{array}\right),
\end{gathered}
$$

where $l_{m}$ is the m-dimensional vector of ones, $P_{\xi \xi, j j}^{(i)}$ are the $m_{j} \times m_{j}$ correlation matrices, and $\phi_{j}^{(i)}, \sigma_{j}^{(i)}$ and $\gamma_{j}^{(i)}$ are scalar parameters. Hereafter, we refer to the model in equations (1), (2), (5) and (6) as the $K$-component Block Structure MSVL (BS-MSVL) model. The number of parameters in the $\mathrm{BS}$ model is $0.5 M(M+1)+3 K B+0.5 K\left\{B(B-1)+\sum_{j=1}^{B} M_{j}\left(M_{j}-1\right)\right\}$.

For practical purpose, we compare the number of parameters in the MSVL, MSVL2C BS-MSVL2C models. When $M=9$ and $B=3(M=50$ and $B=5)$ with the same block size, the number of parameters in the BS-MSVL2C model is 87 (1775). For the MSVL and MSVL2C models for the case $M=9 \quad(M=50)$, they are 108 (2650) and 171 (4025). Thus, the BS-MSV model is parsimonious in terms of the number of parameters.

In empirical analysis, the appropriate number of component is $K=2$ for univariate $\mathrm{SV}$ models, as shown by Alizadeh, Brandt and Diebold (2002) and Chernov et al. (2003). Here, we stress an interpretation of the two-factor model by Shephard (1996). Shephard 
(1996) introduces an approach to deal with permanent and transitory components in stochastic volatility models, as those components in the GARCH specification by Engle and Lee (1993). In the specification, the AR(1) parameter of the permanent component is equal to one, while it is located between -1 and 1 as usual for the transitory component. Inspired by the idea, we suggest the complete BS model for the BS-MSVL2C model, which has the first component with

$$
\phi^{(1)}=\imath_{M}, \quad \sigma_{\xi}^{(1)}=\sigma_{*}^{(1)} l_{M}, \quad \gamma^{(1)}=\gamma_{*}^{(1)} l_{M}, \quad P_{\xi \xi}^{(1)}=I_{M},
$$

where $\sigma_{*}^{(1)}$ and $\gamma_{*}^{(1)}$ are scalar parameters. We refer to the model as the 'CBS' model. The number of parameters in the BS model is $0.5 M(M+1)+3 B+2+0.5\left\{B(B-1)+\sum_{j=1}^{B} M_{j}\left(M_{j}-1\right)\right\}$. When $M=9$ and $B=3$ ( $M=50$ and $B=5$ ) with the same block size, the number of parameters in the CBS model is $68(1527)$.

\section{Estimation}

For the estimation of the above various MSVL models, we estimate the mean and volatility equations separately. Following Asai and McAleer (2009a), we may employ the Monte Carlo likelihood (MCL) approach proposed by Durbin and Koopman (1997), in order to estimate the $K$-component MSVL models. The MCL method is based on the state-space form with non-Gaussian measurement errors. In the MCL method, the likelihood function can be approximated arbitrarily by decomposing it into a Gaussian part, which is constructed by the Kalman filter, and a remainder function, for which the 
expectation is evaluated through simulation.

Regarding the family of SV models, we may have the state space form by the logarithmic transformation of squared returns, as in Harvey, Ruiz and Shephard (1994) for the basic MSV model. By the transformation, we will however lose the information regarding the correlation between $\varepsilon_{t}$ and $\eta_{t}\left(\xi_{t}\right)$. While Harvey and Shephard (1996) suggested an approach to recover the information for the univariate SV model with the leverage effect (SVL), Asai and McAleer (2006) extended it to the MSVL model using the properties of half normal distributions shown by Leone, Nelson and Nottingham (1961) and Elandt (1961). Sandmann and Koopman (1998) applied the MCL method to the univariate SVL model, while Asai and McAleer (2006, 2009a) adapted it for the several kinds of MSVL models.

It should be noted that we may also work with the quasi-maximum likelihood (QML) estimation based on the state space form, as suggested by Harvey, Ruiz and Shephard (1994). The QML estimator is inefficient, but it is still consistent.

For convenience, we use the sample correlation matrix for the initial value for estimating $P_{\varepsilon \varepsilon}$, which has a major part of parameters as $0.5 M(M-1)$.

\section{Empirical Analysis}

In this section, we estimate the MSVL, BS-MSVL2C and CBS models, and compare their out-of-sample forecasts. Three groups of three assets from three different sectors 
$(B=3$ and $M=9)$ are used, namely Chemical, General Financials, and Oil and Gas Producers including (AIR PRDS.\& CHEMS., ROHM \& HAAS, EASTMAN CHEMICALS), (GOLDMAN SACHS GP., LEHMAN BROS.HDG., MERRILL LYNCH \& CO.) and (CHEVRON, EXXON MOBIL, CONOCOPHILLIPS). Please correct them! These assets have been selected from among a small list of the largest companies between each sector on the basis of the correlations between the squared returns. All the selected stocks belong to the large cap segment of the NYSE, and enter the S\&P 500 index. Given the approach followed in the asset selection, intuitively there possibly exist common patterns in the variances. We chose such a selection approach in order to provide an example where the proposed modelling approach may be useful.

The series considered are daily return indices, collected in the sample period 2 January 2000 to 31 December 2010, giving 2865 observations. We chose two kinds of periods before/after the global financial crisis (GFC) in the following way. We fixed the sample size as $T=1500$ for estimation and forecasting. Then we estimate the model based on the dataset for the years 2000-2005, and forecast daily covariances for the year 2006, corresponding to the period before GFC. With respect to the period which covers the GFC, we use the data for the years 2004-2009 for estimating the models, and conduct forecasting daily covariances for the year 2010. We should add that our data may be influenced by the wars in Afghanistan and Iraq and by the increasing trend in oil prices.

In order to develop the conditional mean for each return, we used the following data sets; a set of interest rates (US Treasury bond 3 months, 6 months, 9 months, 1-3 years, 3-5 years, 5-7 years), oil prices, and two dummies (January and Monday). Interest rates 
are in the form of bond indices. Following Ait-Sahalia and Brandt (2001) and Pesaran and Timmerman $(1995,2000)$, we fit the conditional mean returns with the constant term, the lagged return, the contemporaneous dummies, the lagged Oil returns, and the deviations between the returns of the rates (the following differences between bond indices returns: 6 months minus 3 months, 1-3 years minus 6 months, and so on), giving 10 explanatory variables, as follows:

$$
E\left(R_{t} \mid \mathfrak{I}_{t-1}\right)=\beta_{1}+\beta_{2} R_{t-1}+\beta_{3} D_{t}^{J a n}+\beta_{4} D_{t}^{\text {Mon }}+\beta_{5} R_{t}^{\text {Oil }}+\beta_{6} V_{1 t}+\cdots+\beta_{10} V_{5 t} .
$$

The deviations between the rates, $V_{i t}$, can be considered as a proxy for the curvature of the yield curve, and hence may be useful in predicting stock movements.

Table 1 shows the QML estimates for the MSVL model for two kinds of periods. In order to save spaces, the estimates of $P_{\varepsilon \varepsilon}$ and $P_{\eta \eta}$ are omitted. Regarding the period before GFC shown in Table 1(a), the estimates of $\phi_{j}$ are between 0.955 and 0.997 , while the estimates of $\sigma_{\eta, j}$ varies from 0.067 to 0.542 . These values are typical in the empirical analysis of the SV and MSV models. Most of the estimates of $\gamma_{j}$ are negative and significant, indicating the leverage effects. But some are positive and insignificant. Table 1(a) also shows that minimum value of the estimates of $\gamma_{j}$ is -0.166 , implying that the leverage effects are weak or negligible for the datasets. Table 1(b) shows the estimation results for the period including the GFC. Compare to Table 1(a), the estimates of $\phi_{j}, \sigma_{\eta, j}$ and $\gamma_{j}$ are similar. Again, the leverage effects are 
minor. Unlike Table 1(a), some of the estimates of $\sigma_{\varepsilon, j}$ are larger than 2. Also, all the values of the estimates of $\sigma_{\varepsilon, j}$ are larger than those for the period before GFC, showing the increase in unexplained factor.

Table 2 gives the QML estimates for the MSVL2C-BS model. We should note that the results for volatility part are 'block-based' by construction. With respect to the period before GFC, the estimates of $\phi_{j}^{(1)}$ are close one, while those of $\phi_{j}^{(2)}$ are far from one. Also, the estimates of $\sigma_{j}^{(1)}$ are smaller than those of $\sigma_{j}^{(2)}$. These results are typical in the two-component SV and MSV models. The leverage effects by the first component are negative and significant for all three blocks, while one of the second components gives a positive value. Compared with MSVL model, the estimates of $\sigma_{\varepsilon, j}$ are similar. Turing to the period including the GFC, the estimates in Table 2(b) are similar to Table 2(a) except for the leverage effects. In the period, the first and second components show a stronger leverage effects than the period before GFC. The estimates of $\sigma_{\varepsilon, j}$ are smaller than the estimates for the MSVL model, implying that the unexplained factor in the MSVL model for GFC was explained by the second component for some extent.

Table 3 presents the QML estimates for the CBS models, which is specified by setting parameters in the first component to be the same in all blocks such that $\phi_{j}^{(1)}=1$, $\sigma_{j}^{(1)}=\sigma_{*}^{(1)}$ and $\gamma_{j}^{(1)}=\gamma_{*}^{(1)}$. Table 3(a) and Table 3(b) shows that the estimates of $\sigma_{*}^{(1)}$ are larger than the estimates given in Table 2, while the $\gamma_{*}^{(1)}$ in Table 2 is insignificant. According to the specification, the estimates of the second components are different 
from Table 2, but these values are typical in the two component SV and MSV models.

For the reminder part of the section, we calculate the forecasts of VaR thresholds as a diagnostic checking. As explained above, the first period for forecasting is the year 2006 which consists of 260 observations, while the second period is the year 2010, giving 261 observations.

We examine characteristics of stock portfolios which are constructed based on covariance matrix forecasts from the MSVL, BS-MSVL2C and CBS models. As the covariance matrix is defined by $C_{t}=D_{t} P_{\varepsilon \varepsilon} D_{t}$, its one-step-ahead forecasts are given by $\hat{C}_{t}=\hat{D}_{t} \hat{P}_{\varepsilon \varepsilon} \hat{D}_{t}$, where $\hat{D}_{t}$ contains the forecasts of volatility in the diagonal and $\hat{P}_{\varepsilon \varepsilon}$ is the estimated correlation matrix of the conditional distribution for the return. Here, we consider the following three kinds of portfolio. The first one is the minimum variance portfolio (MVP) with the weights given by $w_{t}=\left(\imath_{M}^{\prime} \hat{C}_{t} l_{M}\right)^{-1}\left(\hat{C}_{t} l_{M}\right)$. The second portfolio is the equally weighted portfolio (EWP) with the constant weights of $w_{t}=M^{-1} l_{M}$. The third one is the value-weighted portfolio (VWP) with time-varying weights given by $w_{t}=\left(l_{M}^{\prime}\left(l_{M}+R_{t-1}\right)\right)^{-1}\left(w_{t-1} \circ\left(l_{M}+R_{t-1}\right)\right)$, starting with a EWP at $t=0$.

Given, the portfolio weights, $w_{t}$, we may define the portfolio returns as $R_{p, t}=w^{\prime} R_{t}$. As we assumed the conditional multivariate normal distribution, we have $R_{p, t}=\mu_{p, t}+y_{p, t}$, where $\mu_{p, t}=w_{t}^{\prime} \mu_{t}$ is the conditional mean and $y_{p, t}$ has the conditional normal distribution with mean zero and variance $h_{t}=w_{t}^{\prime} C_{t} w_{t}$. Fixing the 
sample size in estimation to be 1500 , we re-estimate the model and forecast one-step-ahead VaR thresholds for the above two periods. In our analysis, we work with $1 \%$ and $5 \%$ thresholds, i.e. $\hat{\mu}_{t}-1.645 \sqrt{\hat{h}_{t}}$ and $\hat{\mu}_{t}-2.576 \sqrt{\hat{h}_{t}}$, respectively. We define the failure percentage as the ratio of the number of times that the portfolio return exceeds its forecast divided by the number of out-of-sample forecasts.

In addition to the three models, we consider a combined approach based on BS-MSVL2C and CBS models, by choosing the portfolio which gives larger forecasts of portfolio variance. It is expected to adjust the fluctuations on BS and CBS models brought by restricting parameters on the MSVL2C model.

In order to assess the estimated VaR thresholds, the unconditional coverage and independence tests developed by Christoffersen (1998) are widely used. A drawback of the Christoffersen (1998) test for independence is that it tests against a particular alternative of a first-order dependence. The duration-based approach in Christoffersen and Pelletier (2004) allows for testing against more general forms of dependence but still requires a specific alternative. Recently, Candelon et al. (2010) have developed a more robust procedure which does not need a specific distributional assumption for the durations under the alternative. Consider the "hit sequence" of VaR violations, which takes a value of one if the loss is greater than the VaR threshold, and takes the value zero if the $\mathrm{VaR}$ is not violated. If we could predict the $\mathrm{VaR}$ violations, then that information may help to construct a better model. Hence, the hit sequence of violations should be unpredictable, and should follow an independent Bernoulli distribution with parameter $p$, indicating that the duration of the hit sequence should follow a geometric 
distribution

The GMM duration-based test developed by Candelon et al. (2010) works with the J-statistic based on the moments defined by the orthonormal polynomials associated with the geometric distribution. The conditional coverage test and independence test based on $q$ orthnormal polynomials have asymptotic $\chi_{q}^{2}$ and $\chi_{q-1}^{2}$ distributions under their respective null distributions. The unconditional coverage test is given as a special case of the conditional coverage test with $q=1$.

Table 4 gives the test results for three kinds of portfolios based on the MSVL, BS-MSVL2C and CBS models and the combined BS+CBS approach, for the period before GFC. The test statistics for the MSVL model for 5\% VaR thresholds are rejected for all three portfolios. The tests for the BS model are rejected for $5 \%$ and $1 \% \mathrm{VaR}$ thresholds for the minimum variance portfolio. All the results for CBS and BS+CBS passed the tests. Regarding the period after GFC, Table 5 indicates that the tests for the MSVL model are rejected for all three portfolios. For the period, the minimum variance portfolio calculated by the CBS model gave unsatisfactory results. All the results for BS and BS+CBS passed the tests. Hence, the combined BS+CBS approach gives the best results for the forecasts before/after GFC.

\section{Conclusion}

In this paper we present a class of multivariate stochastic volatility models which is nested in the multi-component model with leverage effects suggested by Asai and McAleer (2009a). The distinctive feature of our model is that, contrary to fully 
parameterized MSV models, it remains feasible in moderate to large cross-sectional dimensions. This result is achieved by imposing a block structure on the model parameter matrices. The variables could be grouped by using some economic or financial criteria, or following data-driven classifications. In addition, by the introduction of the blocks, if these have an economic interpretation, the model we propose preserves the interpretation of coefficients, a feature which is generally lost in feasible MSV models.

We present then an empirical application where the proposed model is estimated on a set of US equities, and examine the VaR thresholds for several types of portfolio calculated by covariance forecasts. Unlike the MSV model with leverage effects, the results given by the approach based on the block structure is satisfactory.

Although the specification by the block structure has the certain contribution to reduce the number of parameters, the conditional correlation matrix of return vector still has many parameters. The issue is left for future researches. 


\section{References}

Ait-Sahalia, Y., and M.W. Brandt (2001), "Variable Selection for Portfolio Choice", Journal of Finance, 56, 1297-1351.

Aguilar, O. and M. West (2000), "Bayesian Dynamic Factor Models and Portfolio Allocation”, Journal of Business and Economic Statistics, 18, 338-357.

Alizadeh, S., M. Brandt, and F. Diebold (2002), "Range-Based Estimation of Stochastic Volatility Models", Journal of Finance, 57, 1047-1091.

Andersen, T.G. (1994), "Stochastic Autoregressive Volatility: A Framework for Volatility Modeling”, Mathematical Finance, 4, 75-102.

Asai, M. (2008), "Autoregressive Stochastic Volatility Models with Heavy-Tailed Distributions: A Comparison with Multifactor Volatility Models", Journal of Empirical Finance, 15, 332-341.

Asai, M. (2009), "Bayesian Analysis of Stochastic Volatility Models with Mixture-of-Normal Distributions", Mathematics and Computers in Simulation, 79, 2579-2596.

Asai, M. and M. McAleer (2006), "Asymmetric Multivariate Stochastic Volatility", Econometric Reviews, 25, 453-473.

Asai, M. and M. McAleer (2009a), "Multivariate Stochastic Volatility, Leverage and News Impact Surfaces", Econometrics Journal, 12, 292-309.

Asai, M. and M. McAleer (2009b), "The Structure of Dynamic Correlations in Multivariate Stochastic Volatility Models", Journal of Econometrics, 150, 182-192.

Asai, M., M. McAleer and J. Yu (2006), "Multivariate Stochastic Volatility: A Review”, Econometric Reviews, 25, 145-175.

Billio, M., M. Caporin and M. Gobbo, (2006), "Flexible Dynamic Conditional 
Correlation Multivariate GARCH for Asset Allocation", Applied Financial Economics Letters, 2, 123-130.

Bauwens, L., S. Laurent and J.V.K. Rombouts (2006), "Multivariate GARCH: A Survey", Journal of Applied Econometrics, 21, 79-109.

Bollerslev, T. (1990), "Modelling the Coherence in Short-run Nominal Exchange Rates: A Multivariate Generalized ARCH Approach", Review of Economics and Statistics, 72 , 498-505.

Bollerslev, T., R.F. Engle and J. Woodridge (1988), “A Capital Asset Pricing Model with Time Varying Covariances”, Journal of Political Economy, 96, 116-131.

Calvet, L.E., A.J. Fisher, and S.B. Thompson (2006), "Volatility Comovement: A Multifrequency Approach”, Journal of Econometrics, 131, 179-215.

Candelon, B., G. Colletaz, C. Hurlin, and S. Tokpavi (2010), "Backtesting Value-at-Risk: A GMM Duration-Based Approach", to appear in Journal of Financial Econometrics.

Chan, D., R. Kohn, and C. Kirby (2006), "Multivariate Stochastic Volatility Models with Correlated Errors", Econometric Reviews, 25, 245-274.

Chernov, M., A.R. Gallant, E. Ghysels and G. Tauchen (2003), “Alternative Models for Stock Price Dynamics", Journal of Econometrics, 116, 225-257.

Chib, S., F. Nardari, and N. Shephard (2002), "Markov Chain Monte Carlo Methods for Generalized Stochastic Volatility Models”, Journal of Econometrics, 108, 281-316.

Chib, S., F. Nardari, and N. Shephard (2006), "Analysis of High Dimensional Multivariate Stochastic Volatility Models”, Journal of Econometrics, 134, 341-371.

Chib, S., Y. Omori and M. Asai (2009), "Multivariate Stochastic Volatility", in: T.G. Andersen, R.A. Davis, J.-P. Kreiss and T. Mikosch (eds.), Handbook of Financial Time Series, Springer-Verlag, New York, 365-400. 
Christoffersen, P. (1998), "Evaluating Interval Forecasts," International Economic Review, 39, 841-62.

Christoffersen, P., Jacobs, K. and Y. Wang (2008), "Option Valuation with Long-run and Shortrun Volatility Components”, Journal of Financial Economics, 90, 272-297.

Christoffersen, P. and D. Pelletier (2004), "Backtesting Value-at-Risk: A Duration-Based Approach", Journal of Financial Econometrics, 2, 84-108.

Danielsson, J. (1998), "Multivariate Stochastic Volatility Models: Estimation and a Comparison with VGARCH Models", Journal of Empirical Finance, 5, 155-173.

Ding, Z. and R.F. Engle (2001), "Large Scale Conditional Covariance Matrix Modeling, Estimation and Testing”, Academia Economic Papers, 1, 83-106.

Elandt, R.C. (1961), “The Folded Normal Distribution: Two Methods of Estimating Parameters from Moments", Technometrics, 3, 551-562.

Engle, R.F. (2002), "Dynamic Conditional Correlation: A Simple Class of Multivariate Generalized Autoregressive Conditional Heteroskedasticity Models”, Journal of Business and Economic Statistics, 20, 339-350.

Engle, R.F. and K.F. Kroner (1995), "Multivariate Simultaneous Generalized ARCH”, Econometric Theory, 11, 122-150.

Engle, R.F. and G.G.J. Lee (1993), "A Permanent and Transitory Component Model of Stock Return Volatility”, Discussion Paper 92-44R, University of California, San Diego.

Fleming, J., C. Kirby and B. Ostdiek (2001), "The Economic Value of Volatility Timing”, Journal of Finance, 56, 329-352.

Harvey, A.C., E. Ruiz and N. Shephard (1994), "Multivariate Stochastic Variance Models", Review of Economic Studies, 61, 247-264.

Harvey, A.C. and N. Shephard (1996), "Estimation of an Asymmetric Stochastic 
Volatility Model for Asset Returns", Journal of Business and Economic Statistics, 14, 429-434.

Hull, J. and A. White (1987), "The Pricing of Options on Assets with Stochastic Volatility", Journal of Finance, 42, 281-300.

Koopman, S.J. and E.H. Uspensky (2002), "The Stochastic Volatility in Mean Model: Empirical Evidence from International Stock Markets", Journal of Applied Econometrics, 17, 667-689.

Lehar, A., M. Scheicher and C. Schittenkopf (2002), "GARCH vs. Stochastic Volatility: Option Pricing and Risk Management”, Journal of Banking and Finance, 23, 323-345.

Leone, F.C., L.S. Nelson, and R.B. Nottingham (1961), "The Folded Normal Distribution", Technometrics, 3, 543-550.

Liesenfeld, R. and R.C. Jung (2000), "Stochastic Volatility Models: Conditional Normality Versus Heavy-Tailed Distributions," Journal of Applied Econometrics, 15, 137-160.

Lopes, H.F. and C.M. Carvalho (2007), "Factor stochastic volatility with time varying loadings and Markov switching regimes", Journal of Statistical Planning and Inference, 137, $3082-3091$.

McAleer, M. (2005), "Automated Inference and Learning in Modeling Financial Volatility", Econometric Theory, 21, 232-261.

Pitt, M.K. and N. Shephard (1999), "Time Varying Covariances: A Factor Stochastic Volatility Approach", in J. M. Bernardo, J. O. Berger, A. P. Dawid, and A. F. M. Smith (Eds.), Bayesian Statistics, Volume 6, Oxford University Press, 547-570.

Shephard, N. (1996), "Statistical Aspects of ARCH and Stochastic Volatility", in: D. R. Cox, D. V. Hinkley, and O. E. Barndorff-Nielsen (Eds.), Time Series Models in Econometrics, Finance and Other Fields, Chapman \& Hall, London, 1-67.

Taylor, S.J. (1986), Modelling Financial Time Series, Chichester, Wiley. 
Tse, Y.K. and A.K.C. Tsui, (2002), “A Multivariate GARCH Model with Time-Varying Correlations", Journal of Business and Economic Statistics, 20, 351-362.

Yu, J. and R. Meyer (2006), "Multivariate Stochastic Volatility Models: Bayesian Estimation and Model Comparison”, Econometric Reviews, 25, 361-384. 
Table 1: QML Estimates for MSVL Model

$$
\begin{aligned}
& y_{t}=D_{t} \varepsilon_{t}, \quad D_{t}=\operatorname{diag}\left\{\exp \left(0.5 h_{t}\right)\right\}, \\
& h_{t+1}=\phi \circ h_{t}+\eta_{t}, \quad\left(\varepsilon_{t}^{\prime}, \eta_{t}^{\prime}\right)^{\prime} \sim N(0, S P S), \\
& S=\operatorname{diag}\left\{\left(\sigma_{\varepsilon}^{\prime}, \sigma_{\eta}^{\prime}\right)^{\prime}\right\}, \quad P=\left(\begin{array}{cc}
P_{\varepsilon \varepsilon} & P_{\varepsilon \eta} \\
P_{\eta \varepsilon} & P_{\eta \eta}
\end{array}\right), \quad P_{\eta \varepsilon}=\operatorname{diag}\{\gamma\} .
\end{aligned}
$$

(a) Before GFC

\begin{tabular}{l|c|c|c|c}
\hline & $\phi_{j}$ & $\sigma_{\eta, j}$ & $\gamma_{j}$ & $\sigma_{\varepsilon, j}$ \\
\hline \hline AIR PRDS.\& & 0.9945 & 0.5420 & -0.1660 & 1.3469 \\
CHEMS. & $(0.0984)$ & $(0.0103)$ & $(0.0097)$ & $(0.1064)$ \\
& 0.9888 & 0.1018 & 0.0157 & 1.5317 \\
ROHM \& HAAS & $(0.0969)$ & $(0.0098)$ & $(0.0151)$ & $(0.0925)$ \\
& 0.9825 & 0.0727 & -0.1530 & 1.4156 \\
EASTMAN & $(0.0969)$ & $(0.0050)$ & $(0.0542)$ & $(0.0894)$ \\
CHEMICALS & 0.9926 & 0.0850 & -0.0569 & 1.4160 \\
\hline \hline GOLDMAN & $(0.0969)$ & $(0.0076)$ & $(0.0523)$ & $(0.0714)$ \\
SACHS GP. & 0.9966 & 0.0672 & -0.0252 & 1.4934 \\
LEHMAN & $(0.0969)$ & $(0.0097)$ & $(0.0066)$ & $(0.0761)$ \\
BROS.HDG. & 0.9925 & 0.0995 & 0.0204 & 1.1346 \\
MERRILL & $(0.0969)$ & $(0.0023)$ & $(0.0364)$ & $(0.0715)$ \\
LYNCH \& CO. & 0.9585 & 0.1899 & -0.0523 & 1.3024 \\
\hline \hline & $(0.0235)$ & $(0.0258)$ & $(0.0010)$ & $(0.0311)$ \\
CHEVRON & 0.9804 & 0.1562 & -0.1131 & 1.2565 \\
EXXON MOBIL & $(0.0975)$ & $(0.0531)$ & $(0.0010)$ & $(0.0844)$ \\
CONOCOPHILL & 0.9554 & 0.2069 & 0.0151 & 1.5038 \\
IPS & $(0.0177)$ & $(0.0217)$ & $(0.0298)$ & $(0.0333)$ \\
\hline \hline
\end{tabular}

Note: Standard errors are given in parentheses. The estimates of $P_{\varepsilon \varepsilon}$ and $P_{\eta \eta}$ are omitted to save space. 
Table 1 (Cont.): QML Estimates for MSVL Model

$$
\begin{aligned}
& y_{t}=D_{t} \varepsilon_{t}, \quad D_{t}=\operatorname{diag}\left\{\exp \left(0.5 h_{t}\right)\right\}, \\
& h_{t+1}=\phi \circ h_{t}+\eta_{t}, \quad\left(\varepsilon_{t}^{\prime}, \eta_{t}^{\prime}\right)^{\prime} \sim N(0, S P S), \\
& S=\operatorname{diag}\left\{\left(\sigma_{\varepsilon}^{\prime}, \sigma_{\eta}^{\prime}\right)^{\prime}\right\}, \quad P=\left(\begin{array}{cc}
P_{\varepsilon \varepsilon} & P_{\varepsilon \eta} \\
P_{\eta \varepsilon} & P_{\eta \eta}
\end{array}\right), \quad P_{\eta \varepsilon}=\operatorname{diag}\{\gamma\} .
\end{aligned}
$$

(b) Middle and After GFC

\begin{tabular}{l|c|c|c|c}
\hline & $\phi_{j}$ & $\sigma_{\eta, j}$ & $\gamma_{j}$ & $\sigma_{\varepsilon, j}$ \\
\hline \hline AIR PRDS.\& & 0.9673 & 0.2364 & -0.0998 & 1.5828 \\
CHEMS. & $(0.0981)$ & $(0.0108)$ & $(0.0248)$ & $(0.4500)$ \\
& 0.9775 & 0.1702 & -0.0496 & 1.8041 \\
ROHM \& HAAS & $(0.0981)$ & $(0.0098)$ & $(0.0248)$ & $(0.6056)$ \\
EASTMAN & 0.9860 & 0.1257 & -0.0636 & 2.2625 \\
CHEMICALS & $(0.0981)$ & $(0.0056)$ & $(0.0251)$ & $(0.6718)$ \\
\hline \hline GOLDMAN & 0.9905 & 0.1452 & -0.0857 & 2.3722 \\
SACHS GP. & $(0.0981)$ & $(0.0101)$ & $(0.0248)$ & $(0.5559)$ \\
LEHMAN & 0.9818 & 0.2068 & -0.0095 & 2.1927 \\
BROS.HDG. & $(0.0981)$ & $(0.0124)$ & $(0.0248)$ & $(0.7367)$ \\
MERRILL & 0.9885 & 0.1731 & -0.1088 & 2.0450 \\
LYNCH \& CO. & $(0.0981)$ & $(0.0092)$ & $(0.0248)$ & $(0.4855)$ \\
\hline \hline CHEVRON & 0.9645 & 0.1481 & -0.1019 & 1.6192 \\
EXXON MOBIL & $(0.0981)$ & $(0.0153)$ & $(0.0249)$ & $(0.1271)$ \\
CONOCOPHILL & 0.9727 & 0.1413 & -0.0738 & 1.5058 \\
IPS & $(0.0982)$ & $(0.0134)$ & $(0.0249)$ & $(0.1059)$ \\
\hline \hline NOte: Stang & 0.1870 & 0.0520 & 1.9209 \\
& $(0.0910)$ & $(0.0111)$ & $(0.0249)$ & $(0.0874)$ \\
\hline
\end{tabular}

Note: Standard errors are given in parentheses. The estimates of $P_{\varepsilon \varepsilon}$ and $P_{\eta \eta}$ are omitted to save space. 
Table 2: QML Estimates for MSVL2C-BS Models

$$
\begin{aligned}
& y_{t}=D_{t} \varepsilon_{t}, \quad D_{t}=\operatorname{diag}\left\{\exp \left(0.5 h_{t}^{(1)}+0.5 h_{t}^{(2)}\right)\right\}, \quad h_{t+1}^{(1)}=\phi^{(1)} \circ h_{t}^{(1)}+\xi_{t}^{(1)}, \quad h_{t+1}^{(2)}=\phi^{(2)} \circ h_{t}^{(2)}+\xi_{t}^{(2)} \text {, } \\
& \left(\varepsilon_{t}^{\prime}, \xi_{t}^{(1)^{\prime}}, \xi_{t}^{(2)^{\prime}}\right)^{\prime} \sim N(0, S P S), \quad S=\operatorname{diag}\left\{\left(\sigma_{\varepsilon}^{\prime}, \sigma_{\xi}^{(1)^{\prime}}, \ldots, \sigma_{\xi}^{(K)^{\prime}}\right)^{\prime}\right\}, \\
& P=\left(\begin{array}{cc}
P_{\varepsilon \varepsilon} & \Gamma \\
\Gamma^{\prime} & P_{\xi \xi}
\end{array}\right), \quad P_{\xi \xi}=\left(\begin{array}{cc}
P_{\xi \xi}^{(1)} & O \\
O & P_{\xi \xi}^{(2)}
\end{array}\right), \quad \Gamma=\left(\begin{array}{ll}
\Gamma_{1} & \Gamma_{2}
\end{array}\right), \quad \Gamma_{i}=\operatorname{diag}\left\{\gamma^{(i)}\right\}, \\
& \phi^{(i)}=\left[\begin{array}{l}
\phi_{1}^{(i)} l_{3} \\
\phi_{2}^{(i)} l_{3} \\
\phi_{3}^{(i)} l_{3}
\end{array}\right], \quad \sigma_{\xi}^{(i)}=\left[\begin{array}{l}
\sigma_{1}^{(i)} l_{3} \\
\sigma_{2}^{(i)} l_{3} \\
\sigma_{3}^{(i)} l_{3}
\end{array}\right], \quad \gamma^{(i)}=\left[\begin{array}{c}
\gamma_{1}^{(i)} l_{3} \\
\gamma_{2}^{(i)} l_{3} \\
\gamma_{3}^{(i)} l_{3}
\end{array}\right], \quad P_{\xi \xi}^{(i)}=\left(\begin{array}{ccc}
P_{\xi \xi, 11}^{(i)} & \rho_{21}^{(i)} l_{3} l^{\prime} & \rho_{31}^{(i)} l_{3} l_{3}^{\prime} \\
\rho_{21}^{(i)} l_{3} l_{3}^{\prime} & P_{\xi \xi, 22}^{(i)} & \rho_{32}^{(i)} l_{3} l_{3}^{\prime} \\
\rho_{31}^{(i)} l_{3} l_{3}^{\prime} & \rho_{32}^{(i)} l_{3} l_{3}^{\prime} & P_{\xi \xi, 33}^{(i)}
\end{array}\right) .
\end{aligned}
$$

\begin{tabular}{|c|c|c|c|c|c|c|c|}
\hline & $\phi_{j}^{(1)}$ & $\sigma_{j}^{(1)}$ & $\gamma_{j}^{(1)}$ & $\phi_{j}^{(2)}$ & $\sigma_{\xi, j}^{(2)}$ & $\gamma_{j}^{(2)}$ & $\sigma_{\varepsilon, j}$ \\
\hline AIR PRDS.\& CHEMS. & & & & & & & $\begin{array}{c}1.4758 \\
(0.2276)\end{array}$ \\
\hline ROHM \& HAAS & $\begin{array}{c}0.9942 \\
(0.0022)\end{array}$ & $\begin{array}{c}0.0460 \\
(0.0018)\end{array}$ & $\begin{array}{l}-0.0141 \\
(0.0021)\end{array}$ & $\begin{array}{c}0.5690 \\
(0.0022)\end{array}$ & $\begin{array}{c}0.5172 \\
(0.0020)\end{array}$ & $\begin{array}{c}-0.0033 \\
(0.0049)\end{array}$ & $\begin{array}{c}1.5442 \\
(0.4829)\end{array}$ \\
\hline EASTMAN CHEMICALS & & & & & & & $\begin{array}{c}1.3937 \\
(0.2205)\end{array}$ \\
\hline GOLDMAN SACHS GP. & & & & & & & $\begin{array}{c}1.5660 \\
(0.2141)\end{array}$ \\
\hline LEHMAN BROS.HDG. & $\begin{array}{c}0.9971 \\
(0.0022)\end{array}$ & $\begin{array}{c}0.0576 \\
(0.0018)\end{array}$ & $\begin{array}{l}-0.0703 \\
(0.0021)\end{array}$ & $\begin{array}{c}0.0352 \\
(0.0022)\end{array}$ & $\begin{array}{c}0.6390 \\
(0.0021)\end{array}$ & $\begin{array}{c}0.0046 \\
(0.0022)\end{array}$ & $\begin{array}{c}1.5238 \\
(0.2634)\end{array}$ \\
\hline MERRILL LYNCH \& CO. & & & & & & & $\begin{array}{r}1.1648 \\
(0.1968) \\
\end{array}$ \\
\hline CHEVRON & & & & & & & $\begin{array}{c}1.3075 \\
(0.2255)\end{array}$ \\
\hline EXXON MOBIL & $\begin{array}{c}0.9836 \\
(0.0022)\end{array}$ & $\begin{array}{c}0.0977 \\
(0.0018)\end{array}$ & $\begin{array}{l}-0.0062 \\
(0.0021)\end{array}$ & $\begin{array}{c}0.3546 \\
(0.0022)\end{array}$ & $\begin{array}{c}0.3754 \\
(0.0025)\end{array}$ & $\begin{array}{l}-0.0389 \\
(0.0061)\end{array}$ & $\begin{array}{c}1.2962 \\
(0.2652)\end{array}$ \\
\hline CONOCOPHILLIPS & & & & & & & $\begin{array}{c}1.5412 \\
(0.3254)\end{array}$ \\
\hline
\end{tabular}

(a) Before GFC

Note: Standard errors are given in parentheses. The estimates of $P_{\varepsilon \varepsilon}, P_{\xi \xi}^{(1)}$ and $P_{\xi \xi}^{(2)}$ are omitted to save space. 
Table 2 (Cont.): QML Estimates for MSVL2C-BS Models

$$
\begin{aligned}
& y_{t}=D_{t} \varepsilon_{t}, \quad D_{t}=\operatorname{diag}\left\{\exp \left(0.5 h_{t}^{(1)}+0.5 h_{t}^{(2)}\right)\right\}, \quad h_{t+1}^{(1)}=\phi^{(1)} \circ h_{t}^{(1)}+\xi_{t}^{(1)}, \quad h_{t+1}^{(2)}=\phi^{(2)} \circ h_{t}^{(2)}+\xi_{t}^{(2)} \text {, } \\
& \left(\varepsilon_{t}^{\prime}, \xi_{t}^{(1)^{\prime}}, \xi_{t}^{(2)^{\prime}}\right)^{\prime} \sim N(0, S P S), \quad S=\operatorname{diag}\left\{\left(\sigma_{\varepsilon}^{\prime}, \sigma_{\xi}^{(1)^{\prime}}, \ldots, \sigma_{\xi}^{(K)^{\prime}}\right)^{\prime}\right\}, \\
& P=\left(\begin{array}{cc}
P_{\varepsilon \varepsilon} & \Gamma \\
\Gamma^{\prime} & P_{\xi \xi}
\end{array}\right), \quad P_{\xi \xi}=\left(\begin{array}{cc}
P_{\xi \xi}^{(1)} & O \\
O & P_{\xi \xi}^{(2)}
\end{array}\right), \quad \Gamma=\left(\begin{array}{ll}
\Gamma_{1} & \Gamma_{2}
\end{array}\right), \quad \Gamma_{i}=\operatorname{diag}\left\{\gamma^{(i)}\right\}, \\
& \phi^{(i)}=\left[\begin{array}{l}
\phi_{1}^{(i)} l_{3} \\
\phi_{2}^{(i)} l_{3} \\
\phi_{3}^{(i)} l_{3}
\end{array}\right], \quad \sigma_{\xi}^{(i)}=\left[\begin{array}{l}
\sigma_{1}^{(i)} l_{3} \\
\sigma_{2}^{(i)} l_{3} \\
\sigma_{3}^{(i)} l_{3}
\end{array}\right], \quad \gamma^{(i)}=\left[\begin{array}{c}
\gamma_{1}^{(i)} l_{3} \\
\gamma_{2}^{(i)} l_{3} \\
\gamma_{3}^{(i)} l_{3}
\end{array}\right], \quad P_{\xi \xi}^{(i)}=\left(\begin{array}{ccc}
P_{\xi \xi, 11}^{(i)} & \rho_{21}^{(i)} l_{3} l^{\prime} & \rho_{31}^{(i)} l_{3} l_{3}^{\prime} \\
\rho_{21}^{(i)} l_{3} l_{3}^{\prime} & P_{\xi \xi, 22}^{(i)} & \rho_{32}^{(i)} l_{3} l_{3}^{\prime} \\
\rho_{31}^{(i)} l_{3} l_{3}^{\prime} & \rho_{32}^{(i)} l_{3} l_{3}^{\prime} & P_{\xi \xi, 33}^{(i)}
\end{array}\right) .
\end{aligned}
$$

\begin{tabular}{|c|c|c|c|c|c|c|c|}
\hline & $\phi_{j}^{(1)}$ & $\sigma_{j}^{(1)}$ & $\gamma_{j}^{(1)}$ & $\phi_{j}^{(2)}$ & $\sigma_{\xi, j}^{(2)}$ & $\gamma_{j}^{(2)}$ & $\sigma_{\varepsilon, j}$ \\
\hline AIR PRDS.\& CHEMS. & & & & & & & $\begin{array}{c}1.2065 \\
(0.1414)\end{array}$ \\
\hline \multirow[t]{2}{*}{ ROHM \& HAAS } & 0.9941 & 0.1129 & -0.1329 & 0.4167 & 0.5837 & -0.0378 & 1.3868 \\
\hline & $(0.0089)$ & $(0.0052)$ & $(0.0088)$ & $(0.0023)$ & $(0.1438)$ & $(0.0011)$ & $(0.1643)$ \\
\hline EASTMAN CHEMICALS & & & & & & & $\begin{array}{r}1.6350 \\
(0.1798) \\
\end{array}$ \\
\hline GOLDMAN SACHS GP. & & & & & & & $\begin{array}{c}1.0506 \\
(0.9409)\end{array}$ \\
\hline \multirow[t]{2}{*}{ LEHMAN BROS.HDG. } & 0.9954 & 0.0927 & -0.0243 & 0.3785 & 0.0010 & -0.0782 & 1.3507 \\
\hline & $(0.0088)$ & $(0.0005)$ & $(0.0025)$ & $(0.0056)$ & $(0.0004)$ & $(0.0039)$ & $(0.1640)$ \\
\hline MERRILL LYNCH \& CO. & & & & & & & $\begin{array}{r}1.4329 \\
(0.1311) \\
\end{array}$ \\
\hline CHEVRON & & & & & & & $\begin{array}{c}1.4919 \\
(0.1756)\end{array}$ \\
\hline EXXON MOBIL & 0.9822 & 0.1036 & -0.3987 & 0.3448 & 0.0024 & -0.0569 & 1.3398 \\
\hline & $(0.0139)$ & $(0.0005)$ & $(0.0063)$ & $(0.0050)$ & $(0.0006)$ & $(0.0065)$ & $(0.2199)$ \\
\hline CONOCOPHILLIPS & & & & & & & $\begin{array}{c}1.6691 \\
(0.0547)\end{array}$ \\
\hline
\end{tabular}

(b) Middle and After GFC

Note: Standard errors are given in parentheses. The estimates of $P_{\varepsilon \varepsilon}, P_{\xi \xi}^{(1)}$ and $P_{\xi \xi}^{(2)}$ are omitted to save space. 
Table 3: QML Estimates for CBS Models

$$
\begin{aligned}
& y_{t}=D_{t} \varepsilon_{t}, \quad D_{t}=\operatorname{diag}\left\{\exp \left(0.5 h_{t}^{(1)}+0.5 h_{t}^{(2)}\right)\right\}, \quad h_{t+1}^{(1)}=\phi^{(1)} \circ h_{t}^{(1)}+\xi_{t}^{(1)}, \quad h_{t+1}^{(2)}=\phi^{(2)} \circ h_{t}^{(2)}+\xi_{t}^{(2)}, \\
& \left(\varepsilon_{t}^{\prime}, \xi_{t}^{(1)^{\prime}}, \xi_{t}^{(2)^{\prime}}\right)^{\prime} \sim N(0, S P S), \quad S=\operatorname{diag}\left\{\left(\sigma_{\varepsilon}^{\prime}, \sigma_{\xi}^{(1)^{\prime}}, \ldots, \sigma_{\xi}^{(K)^{\prime}}\right)^{\prime}\right\}, \quad P=\left(\begin{array}{cc}
P_{\varepsilon \varepsilon} & \Gamma \\
\Gamma^{\prime} & P_{\xi \xi}
\end{array}\right), \quad P_{\xi \xi}=\left(\begin{array}{cc}
P_{\xi \xi}^{(1)} & O \\
O & P_{\xi \xi}^{(2)}
\end{array}\right) \text {, } \\
& \Gamma=\left(\begin{array}{ll}
\Gamma_{1} & \Gamma_{2}
\end{array}\right), \quad \Gamma_{i}=\operatorname{diag}\left\{\gamma^{(i)}\right\}, \quad \phi^{(1)}=l_{9}, \quad \sigma_{\xi}^{(1)}=\sigma_{*}^{(1)} l_{9}, \quad \gamma^{(1)}=\gamma_{*}^{(1)} l_{9}, \quad P_{\xi \xi}^{(1)}=I_{9}, \\
& \phi^{(2)}=\left[\begin{array}{l}
\phi_{1}^{(2)} l_{3} \\
\phi_{2}^{(2)} l_{3} \\
\phi_{3}^{(2)} l_{3}
\end{array}\right], \quad \sigma_{\xi}^{(2)}=\left[\begin{array}{l}
\sigma_{1}^{(2)} l_{3} \\
\sigma_{2}^{(2)} l_{3} \\
\sigma_{3}^{(2)} l_{3}
\end{array}\right], \quad \gamma^{(2)}=\left[\begin{array}{l}
\gamma_{1}^{(2)} l_{3} \\
\gamma_{2}^{(2)} l_{3} \\
\gamma_{3}^{(2)} l_{3}
\end{array}\right], \quad P_{\xi \xi}^{(2)}=\left(\begin{array}{ccc}
P_{\xi, 11}^{(2)} & \rho_{21}^{(2)} l_{l^{\prime}} l^{\prime} & \rho_{31}^{(2)} l_{l_{3}^{\prime}}{ }_{3}^{(2)} \\
\rho_{21}^{(2)} l_{3}^{\prime} & P_{\xi \xi, 22}^{(2)} & \rho_{32}^{(2)} l_{l} l_{3}^{\prime} \\
\rho_{31}^{(2)} l_{3} l_{3}^{\prime} & \rho_{32}^{(2)} l_{3} l_{3}^{\prime} & P_{\xi \xi, 33}^{(2)}
\end{array}\right) \text {. }
\end{aligned}
$$

\begin{tabular}{|c|c|c|c|c|c|c|}
\hline & $\sigma_{*}^{(1)}$ & $\gamma_{*}^{(1)}$ & $\phi_{j}^{(2)}$ & $\sigma_{\xi, j}^{(2)}$ & $\gamma_{j}^{(2)}$ & $\sigma_{\varepsilon, j}$ \\
\hline AIR PRDS.\& CHEMS. & \multirow{9}{*}{$\begin{array}{c}0.3906 \\
(0.1097)\end{array}$} & \multirow{9}{*}{$\begin{array}{l}-0.0097 \\
(0.0361)\end{array}$} & & & & $\begin{array}{c}1.6119 \\
(0.1408)\end{array}$ \\
\hline ROHM \& HAAS & & & $\begin{array}{c}0.7471 \\
(0.0945)\end{array}$ & $\begin{array}{c}0.6037 \\
(0.1259)\end{array}$ & $\begin{array}{c}0.0185 \\
(0.0280)\end{array}$ & $\begin{array}{c}1.5018 \\
(0.1101)\end{array}$ \\
\hline EASTMAN CHEMICALS & & & & & & $\begin{array}{c}1.6204 \\
(0.1976)\end{array}$ \\
\hline GOLDMAN SACHS GP. & & & & & & $\begin{array}{r}1.5833 \\
(0.1413)\end{array}$ \\
\hline LEHMAN BROS.HDG. & & & $\begin{array}{c}-0.3389 \\
(0.2151)\end{array}$ & $\begin{array}{c}0.2565 \\
(0.0561)\end{array}$ & $\begin{array}{c}-0.0224 \\
(0.0853)\end{array}$ & $\begin{array}{c}1.7726 \\
(0.1284)\end{array}$ \\
\hline MERRILL LYNCH \& CO. & & & & & & $\begin{array}{r}1.8617 \\
(0.1336)\end{array}$ \\
\hline CHEVRON & & & & & & $\begin{array}{c}1.6501 \\
(0.1844)\end{array}$ \\
\hline EXXON MOBIL & & & $\begin{array}{c}0.8593 \\
(0.0467)\end{array}$ & $\begin{array}{c}0.0287 \\
(0.0038)\end{array}$ & $\begin{array}{c}-0.0076 \\
(0.0062)\end{array}$ & $\begin{array}{c}1.5601 \\
(0.1102)\end{array}$ \\
\hline CONOCOPHILLIPS & & & & & & $\begin{array}{r}1.5106 \\
(0.1274) \\
\end{array}$ \\
\hline
\end{tabular}

(a) Before GFC

Note: Standard errors are given in parentheses. The estimates of $P_{\varepsilon \varepsilon}$ and $P_{\xi \xi}^{(2)}$ are omitted to save space. 
Table 3 (Cont.): QML Estimates for CBS Models

$$
\begin{aligned}
& y_{t}=D_{t} \varepsilon_{t}, \quad D_{t}=\operatorname{diag}\left\{\exp \left(0.5 h_{t}^{(1)}+0.5 h_{t}^{(2)}\right)\right\}, \quad h_{t+1}^{(1)}=\phi^{(1)} \circ h_{t}^{(1)}+\xi_{t}^{(1)}, \quad h_{t+1}^{(2)}=\phi^{(2)} \circ h_{t}^{(2)}+\xi_{t}^{(2)} \text {, } \\
& \left(\varepsilon_{t}^{\prime}, \xi_{t}^{(1)^{\prime}}, \xi_{t}^{(2)^{\prime}}\right)^{\prime} \sim N(0, S P S), \quad S=\operatorname{diag}\left\{\left(\sigma_{\varepsilon}^{\prime}, \sigma_{\xi}^{(1)^{\prime}}, \ldots, \sigma_{\xi}^{(K)^{\prime}}\right)^{\prime}\right\}, \quad P=\left(\begin{array}{cc}
P_{\varepsilon \varepsilon} & \Gamma \\
\Gamma^{\prime} & P_{\xi \xi}
\end{array}\right), \quad P_{\xi \xi}=\left(\begin{array}{cc}
P_{\xi \xi}^{(1)} & O \\
O & P_{\xi \xi}^{(2)}
\end{array}\right), \\
& \Gamma=\left(\begin{array}{ll}
\Gamma_{1} & \Gamma_{2}
\end{array}\right), \quad \Gamma_{i}=\operatorname{diag}\left\{\gamma^{(i)}\right\}, \quad \phi^{(1)}=\iota_{9}, \quad \sigma_{\xi}^{(1)}=\sigma_{*}^{(1)} l_{9}, \quad \gamma^{(1)}=\gamma_{*}^{(1)} l_{9}, \quad P_{\xi \xi}^{(1)}=I_{9}, \\
& \phi^{(2)}=\left[\begin{array}{l}
\phi_{1}^{(2)} \boldsymbol{l}_{3} \\
\phi_{2}^{(2)} \boldsymbol{l}_{3} \\
\phi_{3}^{(2)} \boldsymbol{l}_{3}
\end{array}\right], \quad \sigma_{\xi}^{(2)}=\left[\begin{array}{l}
\sigma_{1}^{(2)} \boldsymbol{l}_{3} \\
\sigma_{2}^{(2)} \boldsymbol{l}_{3} \\
\sigma_{3}^{(2)} \boldsymbol{l}_{3}
\end{array}\right], \quad \gamma^{(2)}=\left[\begin{array}{l}
\gamma_{1}^{(2)} \boldsymbol{l}_{3} \\
\gamma_{2}^{(2)} \boldsymbol{l}_{3} \\
\gamma_{3}^{(2)} \boldsymbol{l}_{3}
\end{array}\right], \quad P_{\xi \xi}^{(2)}=\left(\begin{array}{ccc}
P_{\xi \xi, 11}^{(2)} & \rho_{21}^{(2)} \boldsymbol{l}_{3} \boldsymbol{l}^{\prime} & \rho_{31}^{(2)} \boldsymbol{l}_{3} \boldsymbol{l}_{3}^{\prime} \\
\rho_{21}^{(2)} \boldsymbol{l}_{3} \boldsymbol{l}_{3}^{\prime} & P_{\xi \xi, 22}^{(2)} & \rho_{32}^{(2)} \boldsymbol{l}_{3} \boldsymbol{l}_{3}^{\prime} \\
\rho_{31}^{(2)} \boldsymbol{l}_{3} \boldsymbol{l}_{3}^{\prime} & \rho_{32}^{(2)} \boldsymbol{l}_{3} \boldsymbol{l}_{3}^{\prime} & P_{\xi \xi, 33}^{(2)}
\end{array}\right) \text {. }
\end{aligned}
$$

\begin{tabular}{|c|c|c|c|c|c|c|}
\hline & $\sigma_{*}^{(1)}$ & $\gamma_{*}^{(1)}$ & $\phi_{j}^{(2)}$ & $\sigma_{\xi, j}^{(2)}$ & $\gamma_{j}^{(2)}$ & $\sigma_{\varepsilon, j}$ \\
\hline AIR PRDS.\& CHEMS. & \multirow{17}{*}{$\begin{array}{c}0.1097 \\
(0.0253)\end{array}$} & \multirow{17}{*}{$\begin{array}{c}0.0206 \\
(0.0389)\end{array}$} & & & & $\begin{array}{c}1.0849 \\
(0.1787)\end{array}$ \\
\hline ROHM \& HAAS & & & 0.9841 & 0.7087 & -0.0010 & 1.0290 \\
\hline & & & $(0.0071)$ & $(0.0879)$ & $(0.0023)$ & $(0.1905)$ \\
\hline EASTMAN CHEMICALS & & & & & & 1.3024 \\
\hline & & & & & & $(0.1631)$ \\
\hline GOLDMAN SACHS GP. & & & & & & 0.7903 \\
\hline & & & & & & $(0.1674)$ \\
\hline \multirow[t]{2}{*}{ LEHMAN BROS.HDG. } & & & -0.0724 & 0.1243 & -0.0160 & 1.1186 \\
\hline & & & $(0.0712)$ & $(0.0281)$ & $(0.0033)$ & $(0.1505)$ \\
\hline \multirow[t]{2}{*}{ MERRILL LYNCH \& CO. } & & & & & & 0.7566 \\
\hline & & & & & & $(0.1784)$ \\
\hline \multirow[t]{2}{*}{ CHEVRON } & & & & & & 0.9880 \\
\hline & & & & & & $(0.1695)$ \\
\hline \multirow[t]{2}{*}{ EXXON MOBIL } & & & 0.9720 & 0.0506 & -0.0047 & 0.9574 \\
\hline & & & $(0.0140)$ & $(0.0044)$ & $(0.0012)$ & $(0.1993)$ \\
\hline \multirow[t]{2}{*}{ CONOCOPHILLIPS } & & & & & & 1.1438 \\
\hline & & & & & & $(0.1803)$ \\
\hline
\end{tabular}

(b) Middle and After GFC

Note: Standard errors are given in parentheses. The estimates of $P_{\varepsilon \varepsilon}$ and $P_{\xi \xi}^{(2)}$ are omitted to save space. 
Table 4: Backtesting VaR Thresholds: Before GFC

(a) Minimum-variance portfolio

\begin{tabular}{cc|cccc}
\hline Model & VaR & $\%$ Violation & UC & ID & CC \\
\hline MSVL & $5 \%$ & 0.0885 & $4.4096 *[0.0357]$ & $0.0922[0.9990]$ & $5.6669[0.3400]$ \\
& $1 \%$ & 0.0192 & $0.5834[0.4450]$ & $1.8953[0.7550]$ & $0.8961[0.9705]$ \\
\hline BS & $5 \%$ & 0.3885 & $79.765 *[0.0000]$ & $8.1793[0.0852]$ & $270.99 *[0.0000]$ \\
& $1 \%$ & 0.3269 & $79.697 *[0.0000]$ & $6.8782[0.1425]$ & $360.03 *[0.0000]$ \\
\hline CBS & $5 \%$ & 0.0769 & $2.7147[0.0994]$ & $0.4148[0.9813]$ & $2.9254[0.7115]$ \\
& $1 \%$ & 0.0192 & $0.8925[0.3448]$ & $4.4728[0.3458]$ & $2.3007[0.8062]$ \\
\hline BS+CBS & $5 \%$ & 0.0769 & $2.7147[0.0994]$ & $0.4148[0.9813]$ & $2.9254[0.7115]$ \\
& $1 \%$ & 0.0192 & $0.8925[0.3448]$ & $4.4728[0.3458]$ & $2.3007[0.8062]$ \\
\hline
\end{tabular}

(b) Equally-weighted portfolio

\begin{tabular}{cc|cccc}
\hline Model & VaR & $\%$ Violation & UC & ID & CC \\
\hline MSVL & $5 \%$ & 0.0808 & $2.7284[0.0986]$ & $13.286 *[0.0099]$ & $14.178[0.0145]$ \\
& $1 \%$ & 0.0192 & $1.7868[0.1813]$ & $2.4639[0.6511]$ & $4.1809[0.5237]$ \\
\hline BS & $5 \%$ & 0.0462 & $0.0691[0.7926]$ & $8.5580[0.1096]$ & $7.5484[0.0731]$ \\
& $1 \%$ & 0.0077 & $0.9701[0.3247]$ & $4.6629[0.3237]$ & $4.5653[0.4712]$ \\
\hline CBS & $5 \%$ & 0.0538 & $0.0032[0.9546]$ & $3.3408[0.5025]$ & $3.1439[0.6778]$ \\
& $1 \%$ & 0.0115 & $1.6182[0.2033]$ & $5.0018[0.2871]$ & $5.4834[0.3598]$ \\
\hline BS+CBS & $5 \%$ & 0.0462 & $0.0691[0.7926]$ & $8.5580[0.1096]$ & $7.5484[0.0731]$ \\
& $1 \%$ & 0.0077 & $0.9701[0.3247]$ & $4.6629[0.3237]$ & $4.5653[0.4712]$ \\
\hline
\end{tabular}

Note: '\% Violation' is the percentage of days when returns are less than the VaR threshold. UC, IND and CC are the GMM duration-base tests for unconditional coverage, independence and conditional coverage, developed by Candelon et al. (2010). The number of orthonormal polynomials is set to 5. $P$-values are in brackets. 
Table 4 (Cont.): Backtesting VaR Thresholds: Before GFC

(c) Value-weighted portfolio

\begin{tabular}{|c|c|c|c|c|c|}
\hline Model & VaR & $\begin{array}{c}\% \\
\text { Violation }\end{array}$ & UC & ID & $\mathrm{CC}$ \\
\hline \multirow[t]{2}{*}{ MSVL } & $5 \%$ & 0.0923 & $4.7616 *[0.0291]$ & $22.794 *[0.0001]$ & $22.036 *[0.0005]$ \\
\hline & $1 \%$ & 0.0192 & $1.7868[0.1813]$ & $2.4639[0.6511]$ & 4.1809 [0.5237] \\
\hline \multirow[t]{2}{*}{$\mathrm{BS}$} & $5 \%$ & 0.0462 & $0.0691[0.7926]$ & 7.5484 [0.1096] & $8.5580[0.1281]$ \\
\hline & $1 \%$ & 0.0115 & $0.2200[0.6390]$ & 0.9373 [0.9192] & 0.7063 [0.9826] \\
\hline \multirow[t]{2}{*}{ CBS } & $5 \%$ & 0.0500 & $0.0561[0.8127]$ & $6.7397[0.1503]$ & 6.7396 [0.2407] \\
\hline & $1 \%$ & 0.0154 & $0.9278[0.3354]$ & $1.3638[0.8505]$ & 1.8259 [0.8727] \\
\hline \multirow[t]{2}{*}{$\mathrm{BS}+\mathrm{CBS}$} & $5 \%$ & 0.0462 & $0.0691[0.7926]$ & $7.5484[0.1096]$ & $8.5580[0.1281]$ \\
\hline & $1 \%$ & 0.0115 & $0.2200[0.6390]$ & 0.9373 [0.9192] & 0.7063 [0.9826] \\
\hline
\end{tabular}

Note: ' $\%$ Violation' is the percentage of days when returns are less than the VaR threshold. UC, IND and CC are the GMM duration-base tests for unconditional coverage, independence and conditional coverage, developed by Candelon et al. (2010). The number of orthonormal polynomials is set to $5 . P$-values are in brackets. 
Table 5: Backtesting VaR Thresholds: After GFC

(a) Minimum-variance portfolio

\begin{tabular}{cc|cccc}
\hline Model & VaR & $\%$ Violation & UC & ID & CC \\
\hline MSVL & $5 \%$ & 0.0881 & $4.3182 *[0.0377]$ & $3.1632[0.5309]$ & $8.4487[0.1332]$ \\
& $1 \%$ & 0.0307 & $4.6064 *[0.0319]$ & $2.9456[0.5670]$ & $12.358 *[0.0302]$ \\
\hline BS & $5 \%$ & 0.0536 & $0.0032[0.9546]$ & $2.5191[0.6412]$ & $2.1080[0.8340]$ \\
& $1 \%$ & 0.0153 & $0.0652[0.7985]$ & $1.1493[0.8864]$ & $0.1935[0.9992]$ \\
\hline CBS & $5 \%$ & 0.0996 & $6.2669 *[0.0123]$ & $1.9365[0.0000]$ & $10.605 *[0.0598]$ \\
& $1 \%$ & 0.0383 & $7.2009 *[0.0073]$ & $4.8976[0.2980]$ & $24.196 *[0.0002]$ \\
\hline BS+CBS & $5 \%$ & 0.0498 & $0.0561[0.8127]$ & $2.4590[0.6520]$ & $2.4836[0.7790]$ \\
& $1 \%$ & 0.0115 & $0.0656[0.4179]$ & $0.6120[0.9617]$ & $0.8500[0.9737]$ \\
\hline
\end{tabular}

(b) Equally-weighted portfolio

\begin{tabular}{cc|cccc}
\hline Model & VaR & $\%$ Violation & UC & ID & CC \\
\hline MSVL & $5 \%$ & 0.1149 & $9.8820 *[0.0017]$ & $1.7100[0.7889]$ & $17.198 *[0.0041]$ \\
& $1 \%$ & 0.0460 & $6.6345 *[0.0100]$ & $6.2647[0.1802]$ & $18.468 *[0.0024]$ \\
\hline BS & $5 \%$ & 0.0498 & $0.0178[0.8940]$ & $4.3026[0.3666]$ & $4.3922[0.4944]$ \\
& $1 \%$ & 0.0115 & $0.7891[0.3744]$ & $0.8451[0.9323]$ & $1.1307[0.9514]$ \\
\hline CBS & $5 \%$ & 0.0575 & $0.1806[0.6708]$ & $7.4071[0.1159]$ & $4.8141[0.4390]$ \\
& $1 \%$ & 0.0077 & $0.8182[0.3657]$ & $3.1751[0.5290]$ & $2.7463[0.7390]$ \\
\hline BS+CBS & $5 \%$ & 0.0460 & $0.2012[0.6538]$ & $2.2051[0.6981]$ & $3.8111[0.5769]$ \\
& $1 \%$ & 0.0077 & $0.8182[0.3657]$ & $3.1751[0.5290]$ & $2.7463[0.7390]$ \\
\hline
\end{tabular}

Note: '\% Violation' is the percentage of days when returns are less than the VaR threshold. UC, IND and CC are the GMM duration-base tests for unconditional coverage, independence and conditional coverage, developed by Candelon et al. (2010). The number of orthonormal polynomials is set to 5. $P$-values are in brackets. 
Table 5 (Cont.): Backtesting VaR Thresholds: After GFC

(c) Value-weighted portfolio

\begin{tabular}{cc|cccc}
\hline Model & & $\begin{array}{c}\% \\
\text { VaR }\end{array}$ & UC & ID & CC \\
& & Violation & & & \\
\hline MSVL & $5 \%$ & 0.1149 & $9.8820 *[0.0017]$ & $1.7100 *[0.0000]$ & $17.198 *[0.0041]$ \\
& $1 \%$ & 0.0498 & $7.5968 *[0.0058]$ & $3.1102[0.5396]$ & $20.345^{*}[0.0011]$ \\
\hline BS & $5 \%$ & 0.0498 & $0.0178[0.8940]$ & $4.3026[0.3666]$ & $4.3922[0.4944]$ \\
& $1 \%$ & 0.0115 & $0.7891[0.3744]$ & $0.8451[0.9323]$ & $1.1307[0.9514]$ \\
\hline CBS & $5 \%$ & 0.0575 & $0.1806[0.6708]$ & $7.4071[0.1159]$ & $4.8141[0.4390]$ \\
& $1 \%$ & 0.0077 & $0.8182[0.3657]$ & $3.1751[0.5290]$ & $2.7463[0.7390]$ \\
\hline CBS & $5 \%$ & 0.0460 & $0.2012[0.6538]$ & $2.2051[0.6981]$ & $3.8111[0.5769]$ \\
& $1 \%$ & 0.0077 & $0.8182[0.3657]$ & $3.1751[0.5290]$ & $2.7463[0.7390]$ \\
\hline
\end{tabular}

Note: ' $\%$ Violation' is the percentage of days when returns are less than the VaR threshold. UC, IND and CC are the GMM duration-base tests for unconditional coverage, independence and conditional coverage, developed by Candelon et al. (2010). The number of orthonormal polynomials is set to $5 . P$-values are in brackets. 